\title{
Trapping and storage of particles in electroactive microwells
}

\author{
Bernardo Cordovez \\ Sibley School of Mechanical and Aerospace Engineering, Cornell University, Ithaca, New York 14853 \\ Demetri Psaltis \\ Department of Electrical Engineering, California Institute of Technology, Pasadena, California 91125 \\ David Erickson ${ }^{\text {a) }}$ \\ Sibley School of Mechanical and Aerospace Engineering, Cornell University, Ithaca, New York 14853
}

(Received 21 September 2006; accepted 7 December 2006; published online 11 January 2007)

\begin{abstract}
The authors describe electroactive microwells which exploit highly localized electrokinetic effects in order to actively concentrate, confine, store, and reject particles in well defined geometries. In this letter the authors present experimental results demonstrating repeatable trapping and repulsion of polystyrene particles in wells ranging in diameter from 6 to $20 \mu \mathrm{m}$ in the presence of a superimposed pressure driven flow. A comprehensive finite element model is developed to describe the transport physics involved in the attraction and repulsion processes. Immediate applications include active cell trapping, particle concentration and unlabeled sensing. (C) 2007 American Institute of Physics. [DOI: 10.1063/1.2430775]
\end{abstract}

The ability to deliver individual or small numbers of particles into confined geometries is of great importance for a number of biological applications (including high throughput pathogen detection, enhanced sensitivity for surface phase binding assays, ${ }^{2}$ parallel single cell trapping, interrogation, and storage ${ }^{3}$ ) and nonbiological applications (including storage of information, directed ${ }^{4}$ or passive $^{5}$ selfassembly processes, and organic electronics $\left.{ }^{6}\right)$. Within this broader set of devices, microwell arrays have become a popular tool for trapping and storing particles as they enable discrete immobilization of cells without the need for surface binding chemistry (as the trapping site geometry is designed to prohibit trapped particles from dislodging easily) in a simple platform compatible with fluorescence microscopy. Such devices enable not only discrete monitoring of small numbers of specimens ${ }^{7}$ but also multiplexed, multiphenotype chemical interrogation without significant cross contamination between closely packed picoliter scale reaction sites. ${ }^{8}$ At present, however, these devices rely largely on passive attraction schemes whereby particles are transported solely by sedimentation, capillary, or hydrodynamic forces, all of which represent inherently slow, indiscriminate, and not easily reversible processes.

Integrated microfluidic devices ${ }^{9}$ enable accurate particle and bioentity transportation by exploiting their electrical, chemical, and optical properties. The mechanisms that have been implemented in these devices include dielectrophoretic (DEP) (see Rosenthal and Voldman ${ }^{10}$ ), magnetic, entropic, optic, and isoelectric trapping. In this letter we present an active trapping architecture compatible with microwell array formats which enables accurate electrokinetic transport, concentration, and rejection of micrometer sized particles in confined geometries. Here we present our initial experiments which demonstrate trapping of $1.9 \mu \mathrm{m}$ diameter polystyrene (PS) beads focusing on developing a description of the fundamental transport physics involved in the trapping and repulsion of the particles. Our approach benefits from the

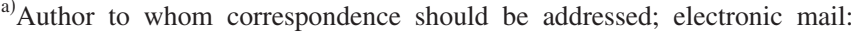
de54@cornell.edu
}

highly localized electric fields that can be generated in electroactive microwells. Since this technique relies on DC electric forces applied on a charged particle, it also enables active repulsion of the trapped targets by reversal of the polarity of the applied field. Although the finite element model presented here is specific to the microwell geometry of interest, the coupling of the electro-osmotic (EO), electrophoretic (EP), and dielectrophoretic transport physics is general to all electrokinetic trapping techniques.

Figure 1(a) shows a schematic of our "electroactive microwells" outlining the basic architecture. The electric field is applied between the upper and lower indium tin oxide (ITO) electrodes, and the wells are defined photolithographically in a polyimide (PI) dielectric. Figure 1(b) is a finite element simulation of the electric field in the well. When a $5 \mathrm{~V} \mathrm{dc}$ bias is applied, the field becomes concentrated at strengths between $10^{5}$ and $10^{6} \mathrm{~V} / \mathrm{m}$ for a $5 \mu \mathrm{m}$ deep well, generating extremely large trapping forces in the well. In a quiescent medium, the trapping stability for such a system can be estimated by comparing the work required to dislodge a particle from the well with $k_{b} T$ via $S=q E h / k_{b} T$ (where $S$ is a non-dimensional stability parameter, $q$ is the charge on the particle, and $h$ is the well height channel height). For the polystyrene particles used here we can compute $S=9 \times 10^{4}$,
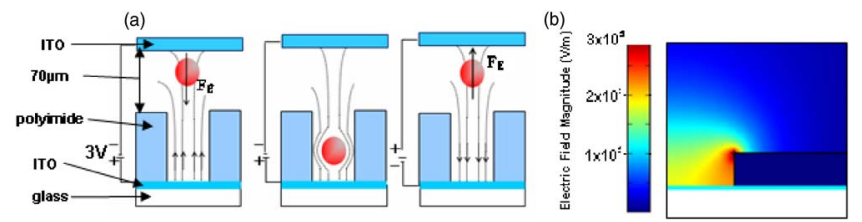

FIG. 1. (Color online) Electroactive microwells: (a) Particles are driven, sensed, and repelled from the well depending on the polarity. Microwells with sizes ranging from 6 to $25 \mu \mathrm{m}$ were patterned on a $5 \mu \mathrm{m}$ thick spin-on photoactive polyimide dielectric on top of a $8 \Omega$ /sq surface resistivity indium tin oxide coated glass slide. An additional ITO electrode was inserted on top of the wells, separated from the polyimide by two $70 \mu \mathrm{m}$ spacers, serving to enclose the channel structure and to close the electric circuit. (b) Axisymmetric finite element simulation displaying strong electric field concentration inside the microwell. Color scheme shows increasing electric field strength from blue (lowest) to red (highest). 

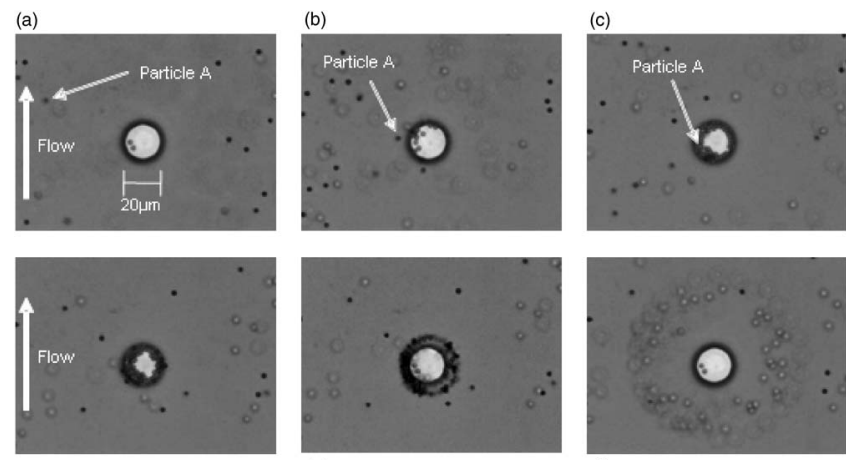

(e)

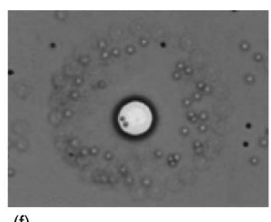

(f)

FIG. 2. Time lapse illustrating particle attraction and repulsion of $1.9 \mu \mathrm{m}$ polystyrene beads in a $20 \mu \mathrm{m}$ well. Conditions are a velocity field of $30 \mu \mathrm{m} / \mathrm{s}$ under a $5 \mathrm{~V}$ potential. (a) Inactive microwell. Note that two particles adhered onto ITO surface before the experiment was performed. (b) Particle attraction begins and beads are drawn into well. (c) Well after $15 \mathrm{~s}$ of attraction. (d) Microwell after $30 \mathrm{~s}$ of attraction. (e) Particles are rejected abruptly as polarity is switched. (f) Ringlike pattern remains and drifts due to superimposed velocity field.

indicating an extremely strong trap ( $q$ was obtained from the electrophoretic mobility measurements from Barker et al. ${ }^{11}$ ). Interestingly, $q$ scales linearly with particle radius, and even for $10 \mathrm{~nm}$ diameter particles $S$ is on the order of 500 .

Figure 2 shows our initial trapping experiments using a $0.1 \mathrm{mM}$ phosphate buffer solution at $p \mathrm{H} 7$ containing $1.9 \mu \mathrm{m}$ diameter polystyrene particles. These particles were carboxyl modified, giving them a negative $\zeta$ potential. It is expected that by applying a positive voltage on the bottom electrode the PS beads will be driven from the bulk flow into the well, and they will be ejected by reversing the polarity. Figure 2 displays time lapse images of particle trapping and rejection in a $20 \mu \mathrm{m}$ well for the case of a $5 \mathrm{~V}$ applied dc bias and an imposed pressure driven flow of $30 \mu \mathrm{m} / \mathrm{s}$ (average velocity). During the attraction phase, Figs. 2(a)-2(d), particles were drawn from the bulk solution into the well over $30 \mathrm{~s}$. Here particles which were either initially positioned or convected into a region approximately three times the diameter of the well would be captured (we refer to this region as the "attraction basin"). However, particles which approached the well in a plane near the surface were observed to be repulsed from it in an upward and radial direction, implying that additional electrokinetic effects aside from the expected electrophoresis have significant impact on the overall attraction process. Figure 2(c) displays how particles tend to collect at the edges of the wells. As a result of this sidewall collection process, specific well occupancy (as a function of volume) was observed to be much higher for smaller wells under experimental conditions. Smaller wells get filled more since field concentration increases with decreasing well diameter, thus generating a stronger trapping force. When the polarity is reversed, Figs. 2(e) and 2(f), particles are repelled out of plane as they approach the well's edge. Particles ejected from the wells form ringlike patterns, suggesting a strong repulsion force that points radially outward from the well's center. The size of the ring shown in Fig. 2(f) roughly coincides with the attraction basin, suggesting that particles decelerate due to both the drop in field intensity away from the well and the contribution of viscous drag. While the time scale to fill the well was dependent on the time required for particle convection into the "attraction Downloaded 12 Jan 2007 to 131.215.240.9. Redistribution subject basin," the rejection time scale was on the order of milliseconds.

A detailed study to characterize the effect of the applied field strength on trapping performance was performed. At the lowest voltage $(1.5 \mathrm{~V})$, particles directly in the vicinity of the wells were drawn in, but generally the electrokinetic trapping force was overcome by the hydrodynamic velocity. As we increased the applied potential to $2 \mathrm{~V}$, weak attraction was obtained. At $2.5 \mathrm{~V}$ strong particle trapping was first observed. As the applied potential was further increased, the electrophoretic velocity began to strongly dominate over the applied pressure driven flow and the diameter of the attraction basin increased. At applied potentials greater than $5 \mathrm{~V}$ the exposed ITO became charred, and electrolysis within the well structure could be observed, representing the highest potential at which the device could be operated. As discussed by Zhu et al. ${ }^{12}$ particle adhesion is of significant concern at small scales. We found that plasma cleaning the ITO wells limited the adhesion of polystyrene onto the wells at low voltages. For voltages above $3 \mathrm{~V}$, some particles remained adhered to the ITO even after polarity reversal [as seen in Fig. 2(e)].

To characterize the coupling and relative strength of the various electrokinetic and convective transport mechanisms, a three-dimensional finite element model of the system was constructed using the microfluidic numerical prototyping techniques described in our previous works. ${ }^{13}$ Briefly the model consists of a simultaneous solution to the constant conductivity electrostatic field $\left(\nabla^{2} \phi=0\right.$, where $\phi$ is the applied potential), Stokes flow $\left(\nabla p-\eta \nabla^{2} v=0\right.$, where $p, \eta$, and $v$ are the pressure, kinematic viscosity, and flow velocity, respectively), and continuity $(\nabla \cdot v=0)$ equations in a domain with identical geometry to that shown in Fig. 2. The solution domain was chosen to be sufficiently large so as to comprise the entire attraction basin. The former of these equations was solved subject to a $3 \mathrm{~V}$ potential difference between the top and bottom electrodes (representing the highest trapping force without particle adhesion) and insulating surfaces ( $\nabla \phi \cdot n=0$, where $n$ is the surface normal) at all other boundaries. The Stokes flow and continuity equations were solved subject to a parabolic velocity profile at the inlet and "apparent slip" condition at the polyimide walls by accounting for their electro-osmotic flow. The slip velocity was computed using the Helmholtz-Smoluchowski relation, $v_{\mathrm{EO}}=\varepsilon \zeta E / \eta$ (where $\varepsilon$ is the permittivity of the medium, $\zeta$ is the zeta potential of the polyimide/solution interface, taken as $-40 \mathrm{mV}$ from Bouriat et al., ${ }^{14}$ and $E=-\nabla \phi$ is the field strength). Free slip conditions were applied at the side boundaries and normal flow $(v \cdot n=0)$ was imposed at the downstream outflow. From this solution the net transport streamlines were computed from the summation of the pressure driven/electro-osmotic flow velocity $\left(v_{\text {flow }}\right)$, electrophoretic velocity $\left(v_{\mathrm{EP}}=\mu_{\mathrm{EP}} E\right)$, where $\mu_{\mathrm{EP}}$ is the electrophoretic mobility of the particles, which was computed to be near $4 \times 10^{-8} \mathrm{~m}^{2} / \mathrm{V}$ s using the zeta potential presented from Barker et al. ${ }^{11}$ and dielectrophoretic velocity $\left(v_{\text {DEP }}\right.$ $=\mu_{\mathrm{DEP}} \nabla E^{2}$ ), where $\mu_{\mathrm{DEP}}$ is the DEP mobility.

Figures 3(a) and 3(b) show the net particle transport lines for this case. Near the edges of the well the lines point radially outwards, resulting mainly from the electro-osmotic transport generated at the polyimide surface. Furthermore, the transport lines form a distinct capture region of roughly to AIP license or copyright, see http://apl.aip.org/apl/copyright.jsp 


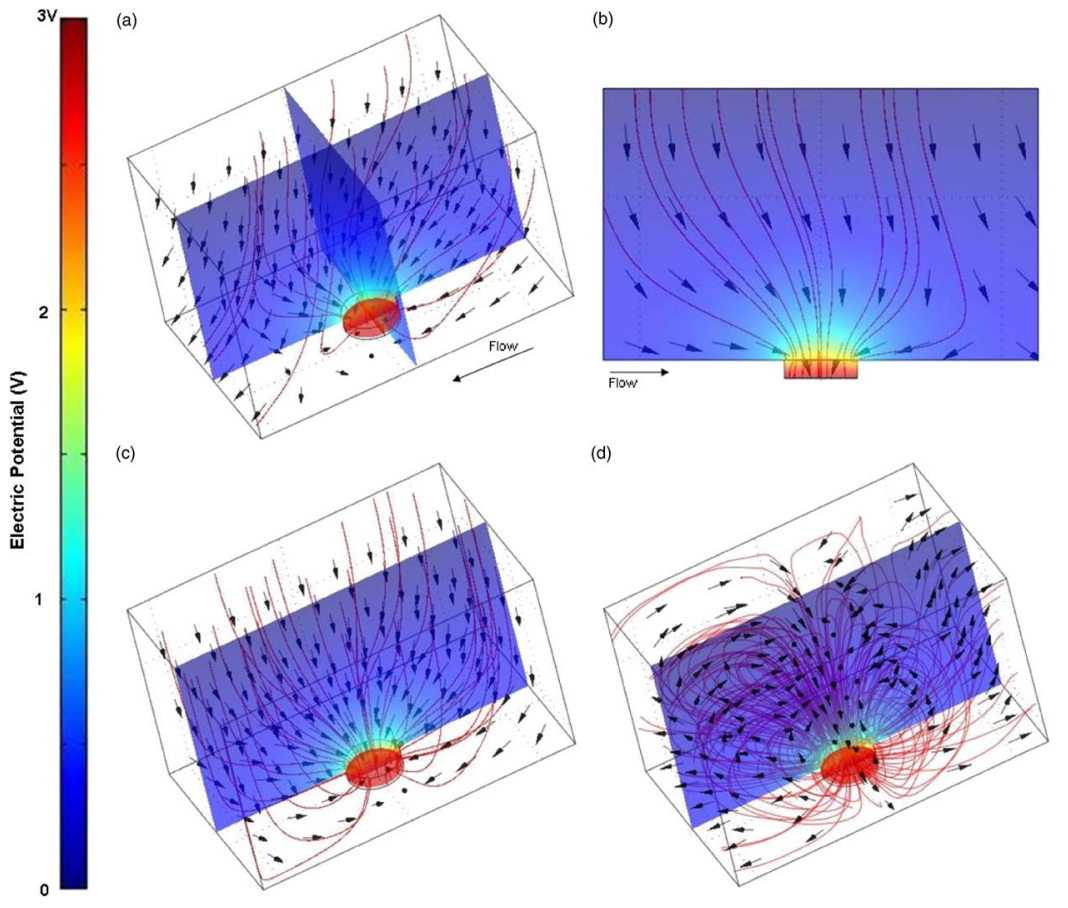

FIG. 3. (Color online) Finite element analysis of electrokinetic effects in $20 \mu \mathrm{m}$ well: (a) Transport lines illustrate competing effects between electro-osmosis, electrophoresis, and dielectrophoresis. (b) Transport lines viewed from cross section. (c) Particle trajectories for charged particles with an uncharged surface. (d) 3D model displaying recirculation induced by dielectric charging. three well diameters wide, consistent with the attraction basin observed experimentally. The transport lines are skewed to the edge farthest downstream from the well due to the contribution of the pressure driven flow. Further simulations conducted at higher electro-osmotic mobility showed clear recirculation near the well's edge (the extreme case of this is shown in Fig. 3(d)) which resulted in a repulsive force on the particles at this location (the local electro-osmotic component acts in the opposite direction of the electrophoretic attraction). This repulsion is consistent with the experimental observations mentioned above for particles resting on the surface near the well, though the effect tended to be much stronger than what was predicted by our simulations. We believe that the EO transport component may have been enhanced in our experimental system due to dielectric charging at the interface, since increasing ion accumulation in the double layer increases the zeta potential at the polyimide/ solution interface. In our model, DEP was found to be small throughout the system except very near the well edges where the field gradients are greatest.

To generalize these observations, we examine how the transport conditions vary as a function of relative contribution of electrokinetic components. The two limiting transport scenarios are presented on Figs. 3(c) and 3(d), respectively. Figure 3(c) displays the case of a charged particle in the presence of an uncharged surface (pure electrophoresis). Figure 3(d) illustrates the recirculatory streamlines that arise in the presence of a charged surface guiding uncharged particles (pure electro-osmosis). In general it was observed that at $\mu_{\mathrm{EP}, \mathrm{PS}} / \mu_{\mathrm{EO}, \mathrm{PI}} \sim 1.5$, there is still noticeable streamline recirculation, while at $\mu_{\mathrm{EP}, \mathrm{PS}} / \mu_{\mathrm{EO}, \mathrm{PI}} \sim 3$ the recirculatory element becomes virtually negligible. Since the EO contribution was generally found to be a hindrance to trapping, a surface treatment could be applied to minimize this component. Although in our current experiment DEP was found to be very small, we expect it to become more significant for well sizes with spatial order below submicrometer dimensions.
In summary, we have demonstrated electroactive microwells which serve to capture, store, and actively repel particles from confined geometries using a combination of electro-osmotic, electrophoretic, and dielectrophoretic effects. The concentration of the electric field within the well enables high trapping potentials which are suitable for confining particles much smaller than those demonstrated here. The highly concentrated electric field within the well also has the potential to enable sensitive impedance based sensing, which could be further enhanced by shrinking the size of the confining geometry down to the same spatial order as that of the target.

This work is supported by National Science Foundation through the Sensors and Sensor Networks program under Grant No. NSF/CTS 0529045 and the DARPA Center for Optofluidic Integration at Caltech.

${ }^{1}$ B. A. Simmons, G. J. McGraw, R. V. Davalos, G. J. Fiechtner, Y. Fintschenko, and E. B. Cummings, MRS Bull. 31, 120 (2006).

${ }^{2}$ M. C. Park, J. Y. Hur, K. W. Kwon, S. H. Park, and K. Y. Suh, Lab Chip 6, 988 (2006).

${ }^{3}$ M. Kang, L. Trofin, M. O. Mota, and C. R. Martin. Anal. Chem. 77, 6243 (2005).

${ }^{4}$ A. Kumar, H. A. Biebuyck, and G. M. Whitesides, Langmuir 10, 1498 (1994).

${ }^{5}$ Y. Cui, M. T. Björk, J. A. Liddle, C. Sönnichsen, B. Boussert, and A. P. Alivisatos, Nano Lett. 4, 1093 (2004).

${ }^{6}$ S. R. Forrest, Nature (London) 428, 911 (2004).

${ }^{7}$ J. R. Rettig and A. Folch, Anal. Chem. 77, 5628 (2005).

${ }^{8}$ A. Khademhosseini, J. Yeh, G. Eng, J. Karp, H. Kaji, J. Borenstein, O. C. Farokhzad, and R. Langer, Lab Chip 5, 1380 (2005).

${ }^{9}$ T. Thorsen, S. J. Maerkl, and S. R. Quake, Science 298, 580 (2002).

${ }^{10}$ A. Rosenthal and J. Voldman, Biophys. J. 88, 2193 (2005).

${ }^{11}$ S. L. R. Barker, M. J. Tarlov, H. Canavan, J. J. Hickman, and L. E. Locascio, Anal. Chem. 72, 4899 (2000).

${ }^{12}$ T. Zhu, S. Zhigang, A. Winkleman, and G. M. Whitesides, Appl. Phys. Lett. 88, 144101 (2006).

${ }^{13}$ D. Erickson, Microfluid. Nanofluid. 1, 301 (2005).

${ }^{14}$ P. Bouriat, P. Saulnier, P. Brochette, A. Graciaa, and J. Lachaise, J. Colloid Interface Sci. 209, 445 (1999). 